\title{
Original
}

\section{New model of stacking layer elements for analyses of reflection and transmission of elastic waves to inhomogeneous layers}

\author{
by
}

\author{
Tadashi $\mathrm{OHyoshI}^{\dagger}$
}

\begin{abstract}
New model of the inhomogeneous layer element is presented for the analysis on a general inhomogeneous elastic layer. Stacking of the new layer elements simulates well a real inhomogeneous layer with small number of the elements. The analytical formulation is made by the transfer matrix method against to the stacked multi-layer structure. Each the stacking layer element is inhomogeneous. Its mechanical impedance varies in linear form with layer thickness.

Applicability of such the new model is inspected with the comparison of the closed form solutions for a linear inhomogeneous layer, with conventional approximate solutions formulated by piling up of many homogeneous layers.

Key Words : Elastic wave, Reflection and Transmissiom, Inhomogeneous Material, Transfer Matrix, Elastic Layer, Mechanical Impedance, New Model.
\end{abstract}

\section{INTRODUCTION}

Studies on reflection and transmission of elastic waves to an inhomogeneous media have been reported by many researchers. The modern attractive problems of inhomogeneous materials are for the functionally gradient solid material. The problems are generally difficult ones to analyze theoretically without resorting to some numerical approach. Most analyses of the papers relating to an inhomogeneous elastic layer may be classified into two kinds of approaches. One is the approximation by piling up large number of homogeneous thin layers, and another one is the theoretical analysis by using restricted function to describe the inhomogeneous property inside a material. The function should be chosen so that the governing equations may have manageable solutions. Therefore, the analysis is liable to go a large amount of numerical calculations or to do for restricted theoretical inhomogeneous materials.

The new model of the element proposed here enables us to solve above the problems. The approach is the use of linear inhomogeneous layer elements instead of the homogeneous elements. The 
inhomogeneity of the element is defined by the variation of mechanical impedance in linear form with the thickness. The combination of the $T$-matrix method and piling the elements up, improves much the adaptability to various real inhomogeneous solid layers with small number of the elements.

Firstly, in order to inspect the adaptability of the model, it has been made computations of the reflection power by closed form solutions for the linear inhomogeneous layer and approximate solutions for the conventional stacking model of homogeneous layers. Comparison of their results

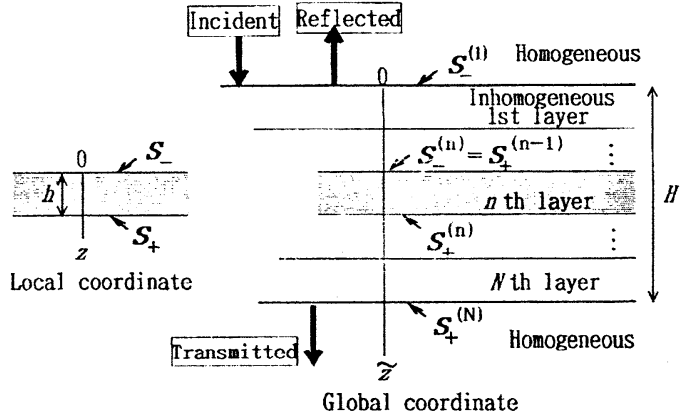

Fig. 1 Stacking geometry of inhomogeneous layers between two homogeneous half spaces. makes clear the advantage of the new model. After then, the reflection and transmission problem of a non-monotonic inhomogeneous layer located between two homogeneous elastic half space, is solved by the new model with the $T$-matrix method, as a demonstrative example.

\section{ANALYSIS}

\section{$T$-matrix for a linear inhomogeneous layer}

Inhomogeneous property of material is specified by the elastic Lamē constants varying as a quadratic function of distance from a layer top surface.

$$
\lambda=\lambda_{0}(1+\boldsymbol{b} \boldsymbol{z})^{2}, \quad \mu=\mu_{0}(1+\boldsymbol{b} \boldsymbol{z})^{2}
$$

$z$ is the local coordinate normal to the layer surface (Fig. 1), and $b$ is a parameter characterizing the inhomogeneous property. Equation(1) is epuivalent that the mechanical impedance variation of the elastic material is linear, provided that the mass density is constant. The constitutive equation for the inhomogeneous layer and the equilibrium equation are

$$
\sigma_{z}=\left(\lambda_{0}+2 \mu_{0}\right)(1+b z)^{2} \frac{\partial \boldsymbol{w}}{\partial \boldsymbol{z}} \quad \text { (2) } \quad \frac{\partial \sigma_{z}}{\partial \boldsymbol{z}}=\rho \frac{\partial^{2} \boldsymbol{w}}{\partial \boldsymbol{t}^{2}}
$$

Consequently, the equation of motion can be written by

$$
\left(b C_{0}\right)^{2} \frac{\partial}{\partial \xi}\left(\xi^{2} \frac{\partial w}{\partial \xi}\right)=\frac{\partial^{2} w}{\partial t^{2}}
$$

in which

$$
\xi=1+b z, \quad C_{0}=\sqrt{\left(\lambda_{0}+2 \mu_{0}\right)} / \rho
$$

The solution of Eq. (4) is

$$
w=\left(A \xi^{\mathrm{m} 1}+B \xi^{\mathrm{m}^{2}}\right) e^{-i \omega t}
$$

where $A$ and $B$ are integral constants, and $m_{1}$ and $m_{2}$ are the roots of

$$
\boldsymbol{m}^{2}+\boldsymbol{m}+\boldsymbol{q}^{2}=\mathbf{0} \quad(7) \quad \boldsymbol{q}=\omega /\left(b \boldsymbol{C}_{0}\right)
$$

That is,

$$
\left\{\begin{array}{l}
m_{1} \\
m_{2}
\end{array}\right\}=\left\{\begin{array}{l}
(-1+\sqrt{ } D) / 2 \\
(-1-\sqrt{ } D) / 2
\end{array}\right\} ; \quad D \geqq 0 \quad \text { or } \quad\left\{\begin{array}{l}
m_{1} \\
m_{2}
\end{array}\right\}=\left\{\begin{array}{l}
(-1+i \sqrt{ } D \mid) / 2 \\
(-1-i \sqrt{ }|D|) / 2
\end{array}\right\} ; \quad D<0
$$

where

$$
D=1-4 q^{2}
$$

The stress corresponding to the displacement (6) is 


$$
\sigma_{z}=\rho C_{0}^{2} \boldsymbol{b} \xi\left(\boldsymbol{m}_{1} \boldsymbol{A} \xi^{n^{1}}+\boldsymbol{m}_{2} \boldsymbol{B} \xi^{\mathrm{m}^{2}}\right) \boldsymbol{e}^{-i \omega t}
$$

Let the boundary conditions at the upper and lower surface of the layer element (Fig. 1) be prescribed as

$$
\begin{array}{lll}
\boldsymbol{w}=\boldsymbol{w}_{-}, & \sigma_{\mathrm{z}}=\sigma_{-} & \text {at } \boldsymbol{z}=\mathbf{0} \\
\boldsymbol{w}=\boldsymbol{w}_{+}, & \sigma_{\mathrm{z}}=\sigma_{+} & \text {at } \boldsymbol{z}=\boldsymbol{h}
\end{array}
$$

Suppressing the time harmonic factor $e^{-i \omega t}$, we have then the representations:

$$
\begin{aligned}
& \left\{\begin{array}{l}
\boldsymbol{w}_{-} \\
\sigma_{-}
\end{array}\right\}=\left(\begin{array}{cc}
1 & 1 \\
\boldsymbol{E}_{-} \boldsymbol{b} \boldsymbol{m}_{1} & \boldsymbol{E}_{-} \boldsymbol{b} \boldsymbol{m}_{2}
\end{array}\right)\left\{\begin{array}{l}
\boldsymbol{A} \\
\boldsymbol{B}
\end{array}\right\} \\
& \left\{\begin{array}{l}
\boldsymbol{w}_{+} \\
\sigma_{+}
\end{array}\right\}=\left(\begin{array}{cc}
\eta_{1} & \eta_{2} \\
\boldsymbol{E}_{+} \boldsymbol{b} \boldsymbol{m}_{1} \eta_{1} & \boldsymbol{E}_{+} \boldsymbol{b} \boldsymbol{m}_{2} \eta_{2}
\end{array}\right)\left\{\begin{array}{l}
\boldsymbol{A} \\
\boldsymbol{B}
\end{array}\right\}
\end{aligned}
$$

in which $\quad \eta_{11}=\exp \left[m_{1} \log (1+b h)\right], \quad \eta_{2}=\exp \left[m_{2} \log (1+b h)\right]$

$$
E_{-}=\rho C_{0}^{2}, \quad \mathbf{E}_{+}=\rho C_{0}^{2}(1+b \boldsymbol{h})
$$

Eliminating $A$ and $B$ from Eq. (14) (15),

$$
\mathcal{S}_{+}=M \mathcal{S}_{-}
$$

where

$$
\boldsymbol{S}_{ \pm}=\left\{\begin{array}{l}
\boldsymbol{w}_{ \pm} \\
\sigma_{ \pm}
\end{array}\right\} \quad \boldsymbol{M}=\left(\begin{array}{ll}
\boldsymbol{M}_{11} & \boldsymbol{M}_{12} \\
\boldsymbol{M}_{21} & \boldsymbol{M}_{22}
\end{array}\right)
$$

and the elements of $T$-matrix $\mathrm{M}$ are

$$
\begin{aligned}
M_{11}=\frac{\boldsymbol{m}_{1} \eta_{2}-\boldsymbol{m}_{2} \eta_{1}}{\boldsymbol{m}_{1}-\boldsymbol{m}_{2}} & \boldsymbol{M}_{12}=\frac{\eta_{1}-\eta_{2}}{\boldsymbol{E}_{-} \boldsymbol{b}\left(\boldsymbol{m}_{1} \boldsymbol{m}_{2}\right)} \\
\boldsymbol{M}_{21}=\frac{-\boldsymbol{E}_{+} \boldsymbol{b} \boldsymbol{m}_{1} \boldsymbol{m}_{2}\left(\eta_{1}-\eta_{2}\right)}{\left(\boldsymbol{m}_{1}-\boldsymbol{m}_{2}\right)} & \boldsymbol{M}_{22}=\frac{\boldsymbol{E}_{+}\left(\boldsymbol{m}_{1} \eta_{1}-\boldsymbol{m}_{2} \eta_{2}\right)}{\boldsymbol{E}_{-}\left(\boldsymbol{m}_{1}-\boldsymbol{m}_{2}\right)}
\end{aligned}
$$

The matrix $M$ has some properties of

$$
\operatorname{det}[M]=1
$$

and all elements $M_{i j}$ is real in spite of the complex roots $m_{j}$, because all the following factors in the matrix elements are always pure imaginary for $D<0$.

$$
\begin{aligned}
& m_{1}-m_{2}=2 i \beta \\
& \eta_{1}-\eta_{2}=2 i e T_{s} \\
& m_{1} \eta_{2}-m_{2} \eta_{1}=2 i e\left(\beta T_{c}-\alpha T_{s}\right) \\
& m_{1} \eta_{1}-m_{2} \eta_{2}=2 i e\left(\beta T_{c}-\alpha T_{s}\right)
\end{aligned}
$$

with replacements

$$
\begin{gathered}
e \equiv \exp [\alpha \log (1+b h)], \\
T_{c} \equiv \cos [\beta \log (1+b h)], \\
T_{s} \equiv \sin [\beta \log (1+b h)]
\end{gathered}
$$

$\alpha$ and $\beta$ stand for real and imagimary part of $m_{1}$ respectively.

Limiting form of $M_{i j}$ for $b \rightarrow 0$ becomes simple expression :

$$
\begin{aligned}
& M_{21}=-Z \omega \sin k h, \quad M_{22}=\cos k h \\
& M_{11}=\cos k h, \quad M_{12}=\frac{\sin k h}{Z \omega} \\
& Z=\rho C_{L}, \quad k=\omega / C_{L}
\end{aligned}
$$

This coincides with $M_{i j}$ for a homogeneous layer. 


\section{Reflection and transmission to inhomogeneous multi-layers}

It is possible to simulate a real inhomogenous layer with piling up the aforementioned linear inhomogeneous layers. The analysis is made for a general inhomogeneous layer which located between two homogeneous elastic half spaces (Fig. 1). Upper and lower boundary values of the layer, $\mathcal{S}_{+}^{(N)}$ and $\mathcal{S}_{-}^{(1)}$, can be related each other by the $T$-matrix. Superscript with parentheses stands for the layer number up to $N$, the total number of the layers. Continuity condition at the interface of $(n)$ th and $(n-1)$ th layer, can be written as

$$
\mathcal{S}_{-}^{(n)}=\mathcal{S}_{+}^{(n-1)} \quad ; n=2, \cdots, N
$$

and by the application of equation (17) for the $n$-th layer element, we have

$$
\mathcal{S}_{+}^{(n)}=\boldsymbol{M}^{(n)} \mathcal{S}_{-}^{(n)} ; n=1, \cdots, N
$$

Eliminating all $\mathcal{S}_{+}^{(n)}$ and $\mathcal{S}_{-}^{(n)}$ except $\mathcal{S}_{-}^{(1)}$ and $\mathcal{S}_{+}^{(N)}$, from Eq. (24) by Eq. (23), we have the relation ;

$$
\boldsymbol{S}_{+}^{(N)}=\widetilde{M} \mathcal{S}_{-}^{(1)}
$$

where

$$
\widetilde{M}=M^{(v)} M^{(N-1)} \cdots M^{(n)}
$$

and

$$
\operatorname{det}[\widetilde{M}]=1
$$

Meanwhile, in both the homogeneous half spaces, the displacement solutions are

$$
\begin{aligned}
& w^{(j)}(\widetilde{z})=A^{(j)} e_{j}^{+}+B^{(j)} e_{j}^{-} \\
& e_{j}^{ \pm}=\exp \left( \pm i \omega \widetilde{z} / C_{L}^{(j)}\right) \quad j=I, I I
\end{aligned}
$$

then the stress representation is

$$
\sigma_{z}^{(j)}(\widetilde{z})=Z^{(j)} i \omega\left(A^{(j)} e_{j}^{+}-B^{(j)} e_{j}^{-}\right)
$$

with mechanical impedance

$$
\boldsymbol{Z}^{(j)}=\rho^{(j)} \boldsymbol{C}_{L^{(j)}}
$$

Of which superscript $j$ takes $I$ and II for the reflected side half space and the transmitted half space respectively. The amplitude $B^{(I)}$ is identically zero because of no reflecting wave generated in the transmitted side. $A^{(1)}$ is the known incident amplitude. From the continuity conditions at both the interfaces assigned by global coordinate $\widetilde{z}=0, \widetilde{z}=H$, we have simultaneous equations for the unknown amplitudes $B^{(\mathrm{I})}$ and $\boldsymbol{A}^{(\mathrm{II})}$.

$$
\begin{aligned}
& \left\{\begin{array}{l}
\boldsymbol{w}^{(\mathrm{I})}(\mathbf{0}) \\
\sigma^{(\mathrm{I})}(\mathbf{0})
\end{array}\right\}=\mathcal{S}_{-}^{(I)}=\left(\begin{array}{cc}
1 & 1 \\
\boldsymbol{Z}^{(\mathrm{I})} \cdot i \omega & -\boldsymbol{Z}^{(\mathrm{I})} i \omega
\end{array}\right)\left\{\begin{array}{l}
A^{(\mathrm{I})} \\
B^{(\mathrm{I})}
\end{array}\right\} \\
& \left\{\begin{array}{c}
\boldsymbol{w}^{(\mathrm{II})}(\boldsymbol{H}) \\
\sigma^{(\mathrm{II})}(\boldsymbol{H})
\end{array}\right\}=\mathcal{S}_{+}^{(N)}=\left(\begin{array}{c}
1 \\
\boldsymbol{Z}^{(\mathrm{II})} i \omega
\end{array}\right) \boldsymbol{A}^{(\mathrm{II})} \exp \left(i \omega / \boldsymbol{C}_{L}{ }^{(\mathrm{II})}\right)
\end{aligned}
$$

After some manipulations of Eqs. (32) (33) with Eq. (25), the coefficients can be obtained as

$$
\begin{aligned}
& R \equiv \frac{\boldsymbol{B}^{(\mathrm{I})}}{\boldsymbol{A}^{(\mathrm{I})}}=\frac{\left(\widetilde{\boldsymbol{M}}_{21}+\boldsymbol{Z}^{(\mathrm{I})} \boldsymbol{i} \omega \widetilde{\boldsymbol{M}}_{22}\right)-\boldsymbol{Z}^{(\mathrm{II})} \boldsymbol{i} \omega\left(\widetilde{\boldsymbol{M}}_{11}+\boldsymbol{Z}^{(\mathrm{I})} \boldsymbol{i} \omega \widetilde{\boldsymbol{M}}_{12}\right)}{-\left(\widetilde{\boldsymbol{M}}_{21}-\boldsymbol{Z}^{(\mathrm{I})} \boldsymbol{i} \omega \widetilde{\boldsymbol{M}}_{22}\right)+\boldsymbol{Z}^{(\mathrm{II})} \boldsymbol{i} \omega\left(\widetilde{\boldsymbol{M}}_{11}-\boldsymbol{Z}^{(\mathrm{I})} \boldsymbol{i} \omega \widetilde{\boldsymbol{M}}_{12}\right)} \\
& T \equiv \frac{A^{(\mathrm{II})}}{\boldsymbol{A}^{(\mathrm{I})}}=\frac{2 \boldsymbol{Z}^{(\mathrm{I})} i \omega \exp \left[-\boldsymbol{i} \omega \boldsymbol{H} / \boldsymbol{C}_{\mathrm{L}}^{(\mathrm{II})}\right]}{-\left(\widetilde{\boldsymbol{M}}_{21}-\boldsymbol{Z}^{(\mathrm{I})} \boldsymbol{i} \omega \widetilde{\boldsymbol{M}}_{22}\right)+\boldsymbol{Z}^{(\mathrm{II})} \boldsymbol{i} \omega\left(\widetilde{\boldsymbol{M}}_{11}-\boldsymbol{Z}^{(\mathrm{I})} \boldsymbol{i} \omega \widetilde{\boldsymbol{M}}_{12}\right)}
\end{aligned}
$$

Now, we define the reflection power coefficient $E_{\mathrm{R}}$ and the transmission power coefficient $E_{T} b y$

$$
\begin{aligned}
\boldsymbol{E}_{R} & =\frac{\boldsymbol{B}^{(\mathrm{I})} \boldsymbol{B}^{(\mathrm{I}) *}}{\boldsymbol{A}^{(\mathrm{I})} \boldsymbol{A}^{(\mathrm{I}) *}} \\
\boldsymbol{E}_{T} & =\frac{\boldsymbol{Z}^{(\mathrm{II})} \boldsymbol{A}^{(\mathrm{II})} \boldsymbol{A}^{(\mathrm{II}) *}}{\boldsymbol{Z}^{(\mathrm{I})} \boldsymbol{A}^{(\mathrm{I})} \boldsymbol{A}^{(\mathrm{I}) *}}
\end{aligned}
$$

where the asterisk ${ }^{*}$ stands for the complex conjugate value. $E_{\mathrm{R}}$ and $\boldsymbol{E}_{\mathrm{T}}$ satisfy the power balance 
law :

$$
E_{\mathrm{R}}+E_{T}=1
$$

This can be confirmed analytically by making use of $\operatorname{det}[\widetilde{M}]=1$ and $\widetilde{M}=\widetilde{M}^{*}$

\section{ADAPTABILITY OF THE MODEL}

To explain adaptability of the model, it is made that the inspection of required number of stacking homogeneous layer elements to approximate adequately the linear inhomogeneous layer by numerical results come from two set of expressions of the power coefficients. The first set of expressions for the linear inhomogeneous layer can be obtained in closed form expressions [1] from Eqs. (36) (37).

They are

$$
\begin{gathered}
\boldsymbol{E}_{\mathrm{R}}= \\
=\frac{1}{\Delta}\left[\left(\boldsymbol{g}_{1}^{2} \boldsymbol{g}_{2}^{2} \boldsymbol{m}_{1}^{2} \boldsymbol{m}_{2}^{2}+1\right)\left(\eta_{1}-\eta_{2}\right)^{2}-2\left\{\left(\boldsymbol{g}_{1}^{2}+\boldsymbol{g}_{2}^{2}\right) \boldsymbol{m}_{1} \boldsymbol{m}_{2}+\boldsymbol{g}_{1} \boldsymbol{g}_{2}\left(\boldsymbol{m}_{1}-\boldsymbol{m}_{2}\right)^{2}\right\} \eta_{1} \eta_{2}\right. \\
\left.+\frac{1}{2}\left\{\left(\boldsymbol{g}_{1}^{2}+\boldsymbol{g}_{2}^{2}\right)\left(\boldsymbol{m}_{1}^{2}+\boldsymbol{m}_{2}^{2}\right)\left(\eta_{1}^{2}+\eta_{2}^{2}\right)-\left(\boldsymbol{g}_{1}^{2}-\boldsymbol{g}_{2}^{2}\right)\left(\boldsymbol{m}_{2}^{2}-\boldsymbol{m}_{2}^{2}\right)\left(\eta_{1}^{2}-\eta_{2}^{2}\right)\right\}\right] \\
\boldsymbol{E}_{\mathrm{T}}=\mathbf{4} \boldsymbol{g}_{1} \boldsymbol{g}_{2}\left(\boldsymbol{m}_{1}-\boldsymbol{m}_{2}\right)^{2} \eta_{1} \eta_{2} / \Delta
\end{gathered}
$$

in which

$$
\begin{gathered}
\Delta=\left(\boldsymbol{g}_{1}^{2} \boldsymbol{g}_{2}^{2} \boldsymbol{m}_{1}{ }^{2} \boldsymbol{m}_{2}{ }^{2}+1\right)\left(\eta_{1}-\eta_{2}\right)^{2}-2\left\{\left(\boldsymbol{g}_{1}{ }^{2}+\boldsymbol{g}_{2}{ }^{2}\right) \boldsymbol{m}_{1} \boldsymbol{m}_{2}-\boldsymbol{g}_{1} \boldsymbol{g}_{2}\left(\boldsymbol{m}_{1}-\boldsymbol{m}_{2}\right)^{2}\right\} \eta_{1} \eta_{2} \\
+\frac{1}{2}\left\{\left(\boldsymbol{g}_{1}{ }^{2}+\boldsymbol{g}_{2}{ }^{2}\right)\left(\boldsymbol{m}_{1}{ }^{2}+\boldsymbol{m}_{2}{ }^{2}\right)\left(\eta_{1}{ }^{2}+\eta_{2}{ }^{2}\right)-\left(\boldsymbol{g}_{1}{ }^{2}-\boldsymbol{g}_{2}{ }^{2}\right)\left(\boldsymbol{m}_{1}{ }^{2}-\boldsymbol{m}_{2}{ }^{2}\right)\left(\eta_{1}{ }^{2}-\eta_{2}{ }^{2}\right)\right\} \\
\boldsymbol{g}_{1}=\frac{\rho \boldsymbol{C}_{0}}{\boldsymbol{Z}^{(\mathrm{I})} \boldsymbol{q}}, \quad \boldsymbol{g}_{2}=\frac{\rho \boldsymbol{C}_{\mathbf{0}}(\mathbf{1}+\boldsymbol{b H})}{\boldsymbol{Z}^{(\mathrm{II})} \boldsymbol{q}} \\
\eta_{\mathbf{K}}=\exp \left[\boldsymbol{m}_{K} \log (\mathbf{1}+\boldsymbol{b H})\right]: \mathbf{K}=\mathbf{1}, \mathbf{2}
\end{gathered}
$$

The another set of expressions for a homogeneous layer between two homogeneous half spaces is

$$
\begin{aligned}
E_{\mathrm{R}} \fallingdotseq & \left\{\left(\gamma_{1}^{2}-1\right)\left(\gamma_{2}^{2}-1\right) \cos \Omega-\left(\gamma_{1}^{2}+1\right)\left(\gamma_{2}^{2}+1\right)+4 \gamma_{1} \gamma_{2}\right\} / \Delta \\
& E_{\mathrm{R}} \fallingdotseq-8 \gamma_{1} \gamma_{2} / \Delta \\
\Delta \fallingdotseq & \left(\gamma_{1}{ }^{2}-1\right)\left(\gamma_{2}{ }^{2}-1\right) \cos \Omega-\left(\gamma_{1}{ }^{2}+1\right)\left(\gamma_{2}{ }^{2}+1\right)-4 \gamma_{1} \gamma_{2}
\end{aligned}
$$

These are obtained analytically from Eqs. (39). (40) by zero limiting operation of inhomogeneous parameter $b$ with the replacements of

$$
\gamma_{1}=\frac{\rho C_{0}}{Z^{(1)}}, \quad \gamma_{2}=\frac{\rho C_{0}}{Z^{(I)}}, \quad \Omega=\frac{2 \omega H}{C_{0}}
$$

Fig. 2 shows coefficients of the reflection power calculated through Eq. (39) and Eq.(44), under the following numerical data conditions. The inhomogeneous parameter $b$ is chosen so that the elastic constans may be continuos through all the media, and mass densities of all the media are identical, $\rho=2[\mathrm{~g} / \mathrm{cc}]$, for simplicity. The minimum wave velocity, $C_{1}$, and the maximum wave velocity, $C_{2}$, are set to $2000[\mathrm{~m} / \mathrm{s}]$ and 6000 $[\mathrm{m} / \mathrm{s}]$ respectively. The solid line is the results for the linear inhomogeneous layer and the dashed lines is the results for the stacked homogeneous layer to simulate the inhomogeneous layer. The

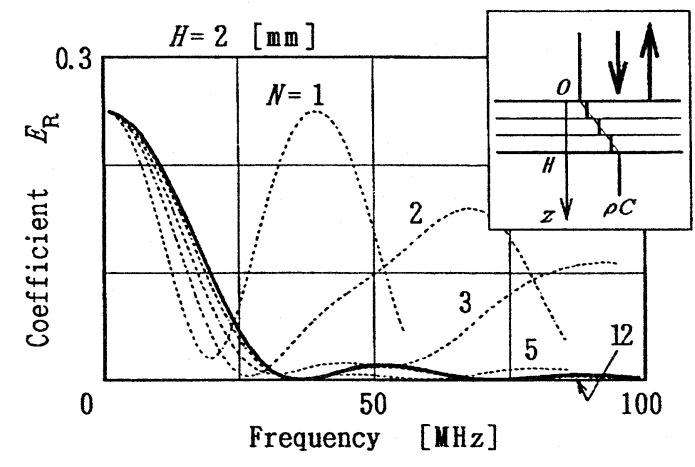

Fig. 2 Required number of staking homogeneous layers to approximate the linear inhomogeneous layer. 
stacking number less then $N=5$ is not available to estimate the reflection and transmission phenomena of the inhomogeneous layer. Consequently, It is expected from Fig. 2 that the usage of the linear inhomogeneous model enables us the considerable reduction of stacking number of layer elements to simulate a real inhomogeneous material. Then the proposed new model has potentials to solve more complex problems for a general inhomogeneous layer with saving computing time.

\section{NUMERICAL EXAMPLES AND REMARKS}

Demonstrative examples of two kinds of inhomogeneous layers are shown in this section. One is the case of monotonic impedance variation (Fig. 3a) and another one is non-monotonic variation (Fig. 3b). The input data of numerical calculation are same to those for Fig. 2. Fig. 4 shows the results of the reflection power coefficients, $E_{R}$, for the monotonic variation to the input frequency with the parameter of thickness $H[\mathrm{~mm}]$. It represents well the property of inhomogeneous transitional layer, that is, the thick layers diminish well the reflection power expectedly in the high

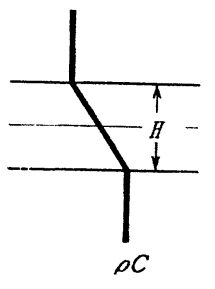

(a) monotonic (b) non-monotonic

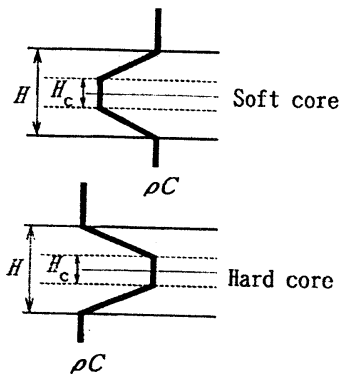

Fig. 3 Variation of the impedance with thickness; monotonic variation (a) and nonmonotonic variation (b)

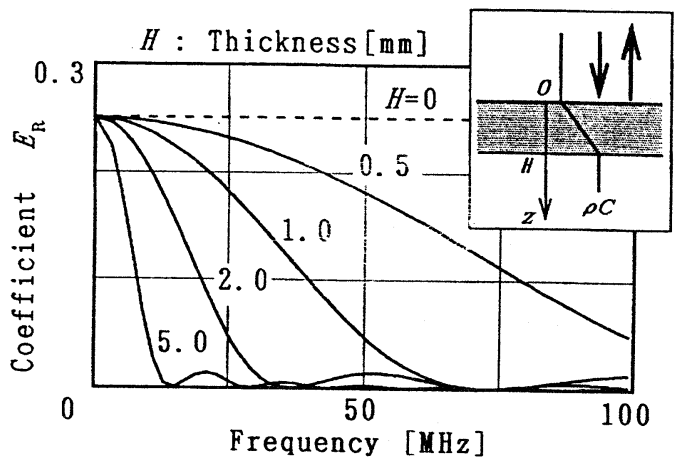

Fig. 4 Coefficient of power reflection to a linear inhomogeneous layer with the parameter of layer thickness $\boldsymbol{H}$

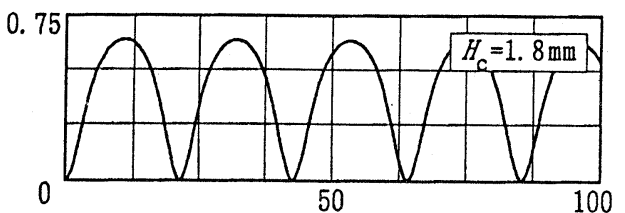

0.75
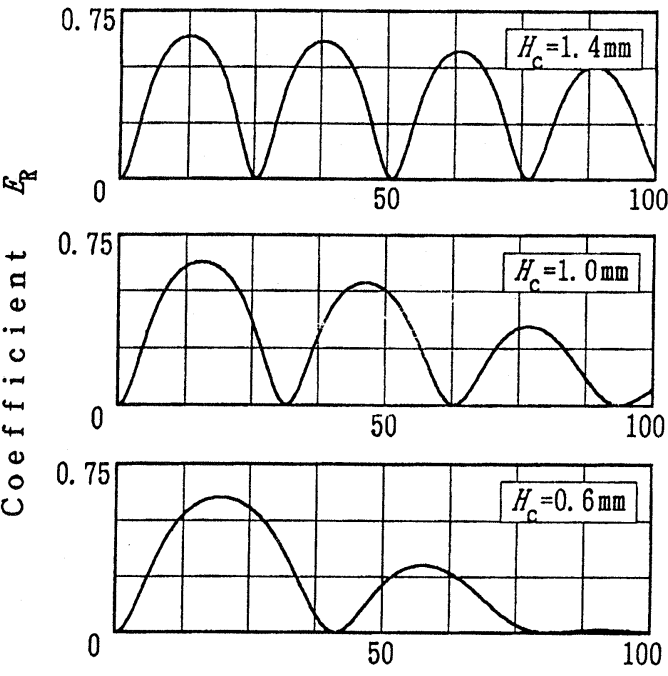

0.75

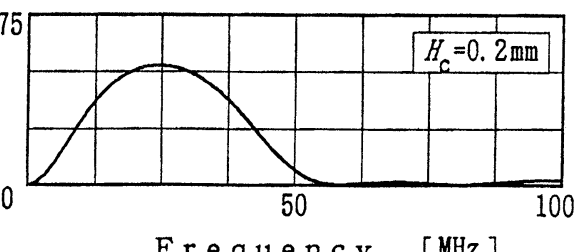

Fig. 5 Coefficient of power reflection to a nonmonotonic inhomogeneous layer having a soft core layer. 
frequency region.

The coefficients $E_{E}$, to another kind of inhomogeneous layers having a homogemeous soft or hard core as illustrated in Fig. $3 \mathrm{~b}$, are also calculated and plotted in Fig. 5 and Fig. 6 to the incident frequency up to $100[\mathrm{MHz}]$. The parameter $H_{c}$ is the core thickness. The total thickness of the layer is set to $2[\mathrm{~mm}]$. They show the small fraction of center core diminishes reflecting power. These numerical calculation can be done very easily.

Application of the new model of stacking inhomogeneous layer element may be possible to analyze conveniently such the wave phenomena for more complex inhomogeneous materials. For oblique incident elastic waves, the analysis also be possible with the potential decomposition method [2] providing the elastic constants of Eq. (1). Analysis on acoustic waves and electromagnetic waves in inhomogeneous media [3] may be instructive to develop the analysis of vector elastic waves.

\section{References}

1) Ohyoshi, T. Proceedings of Conference on Materials and Mechanics, in Kanazawa, Nov. 12, (1991), Vol. A, pp.364-365. (in Japanese)

2) Ewing, W. M., Jardetzky, W. S. and Press, F., "Elastic Waves in Layered Media", Chap. 7., p.328., (1957), McGraw-Hill, New York.

3) Brekhovskikh, L. M. "Waves in Layered Media", (Translated from Russian by Lieberman, D. and edited by Beyer, R. T.), Chap. 3, p.168, (1960), Academic press, New York.
0.75
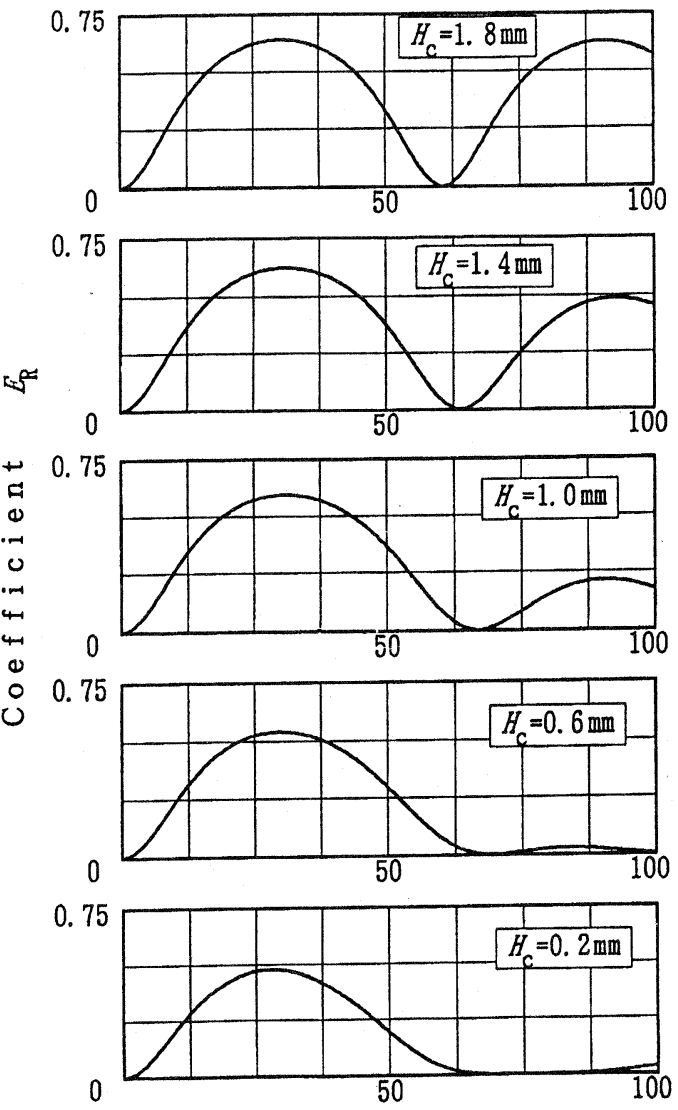

Frequency [ $\mathrm{MHz}]$

Fig. 6 Coefficient of power reflection to a nonmonotonic inhomogeneous layer having a hard core layer. 